\title{
EARLY PHOTOGRAPHIC OBSERVATIONS OF RED AND INFRA- RED FEATURES IN THE SPECTRUM OF SN 1972e
}

\author{
(Abstract) \\ M. F. McCARTHY, S.J. \\ Vatican Observatory, Vatican City State
}

When news of Kowal's discovery (1972) of SN 1972e in NGC 5253 was received at Cerro Tololo on 19 May 1972, an infrared plate was obtained with the Curtis Schmidt and its objective prism which revealed the presence of a faint emission feature between $8600 \AA$ and $8700 \AA$. This was a Kodak IN plate, unhypersensitised and exposed for $25^{m}$ without a filter. On 21 May an exposure of $90^{m}$ was made on an ammonia-sensitized IN plate through a Wratten filter 89B. The Michigan combination objective prism of $10^{\circ}$ with a reciprocal dispersion near $7590 \AA$ of $570 \AA \mathrm{mm}^{-1}$ was used, and the prominent emission-like feature centered at $8650 \AA$ was confirmed.

The Schmidt results together with other spectrographic and photometric observations made at Cerro Tololo near maximum phase were reported by Osmer et al. (1972). Discussion of the $8650 \AA$ feature and its possible identification with the $\mathrm{Ca}$ II triplet at $8498 \AA, 8542 \AA, 8662 \AA$, with O I at $8446 \AA$ or with $\mathrm{N}_{\mathrm{I}} 8629 \AA$ and $8683 \AA$ was presented by McCarthy and Araya (1973) and by McCarthy (1973a, b). Further confirmation of this emission-like feature was obtained by image tube observations made later at Asiago by Ciatti (1973). The identification of the $8650 \AA$ feature with the Ca triplet based on a long series of spectrum scanner observations at Palomar has been given by Searle (1973) and later by Kirshner (1974), and here by Mustel (1974). Herbig (1972) first photographed the deep depression near $8250 \AA$ in SN $1972 \mathrm{e}$ and this was confirmed in our Cerro Tololo observations. This feature is similar to another deep depression noted in the red at $6175 \AA$ by Ford and Rubin in type I supernovae $(1967,1968)$ and also observed in SN 1972e by Herbig (1972) and by McCarthy (1973a). Our objective prism spectra show the following features in the red and the near infrared spectra of SN 1972e near maximum phase:

\begin{tabular}{llll}
$\begin{array}{l}\text { Emission-like } \\
\text { feature }\end{array}$ & $\begin{array}{l}\text { Suggested } \\
\text { sources }\end{array}$ & $\begin{array}{l}\text { Deep depression } \\
\text { feature }\end{array}$ & $\begin{array}{l}\text { Wavelength } \\
\text { difference }\end{array}$ \\
\hline $8650 \AA$ & Ca II, N I, O I & $8250 \AA$ & $-400 \AA$ \\
$6550 \AA$ & $\mathrm{H}, \mathrm{N}$ II & $6175 \AA$ & $-375 \AA$ \\
\hline
\end{tabular}

The association of strong emission-like features with the very deep depression some $400 \AA$ to shortward wavelengths in both red and near infrared regions are the outstanding features present on these objective prism spectra obtained near maxmium phase. The long series of spectra discussed by Searle and Kirschner and by Mustel 
will be decisive in establishing at long last the mechanism responsible for the strong and strange features of type I supernovae spectra.

\section{References}

Ciatti, F.: 1973, Astron. Astrophys. 22, 465.

Ford, W. K. and Rubin, V. C.: 1968, Publ. Astron. Soc. Pacific 80, 466.

Herbig, G. H.: 1972, Circular IAU, No. 2407.

Kirshner, R. P.: 1974, in G. Contopoulos (ed.), Highlights of Astronomy, Vol. 3, D. Reidel Publ. Co., Dordrecht, p. 533.

Kowal, C.: 1972, Circular IAU, No. 2405.

McCarthy, M. F. and Araya, G.: 1973, Bull. Am. Astron. Soc. 5, 12.

McCarthy, M. F.: 1973a, in C. Batalli-Cosmovici (ed.), Internatl. Conf. on Supernovae, University of Lecce (May 1973), in press.

McCarthy, M. F.: 1973b, Ric. Astron. Spec. Vat. 8, No. 21, in press.

Mustel, E.: 1974, this volume, p. 172.

Osmer, P. S., Hesser, J. E., Kunkel, W. E., Lasker, B. M., McCarthy, M. F., and Landolt, A. U.: 1972, Nature Phys. Sci. 238, 21.

Searle, L.: 1973, in C. Batalli-Cosmovici (ed.), Internatl. Conf. on Supernovae, University of Lecce (May 1973), in press. 